\title{
Effect of Sodium Butyrate on Working Memory and Serum Level Expression of Neural Growth Factor in an Animal Model of Prenatal Stress
}

\author{
Forough Akrami ${ }^{1,2}$, Mohammad Amin Edalatmanesh ${ }^{1^{*}}$ \\ ${ }^{1}$ Department of Physiology, College of Sciences, Shiraz Branch, Islamic Azad University, Shiraz, Iran \\ ${ }^{2}$ Department of Physiology, College of Sciences, Fars Sciences and Research Branch, Islamic Azad University, Fars, Iran
}

\section{A BSTRACT}

Introduction: Feeling stressed is common during pregnancy and some pregnant women experience some level of anxiety. Prenatal Stress alters the activity of the hypothalamic-pituitaryadrenal axis of infants and affects glucocorticoid receptors in the hippocampus. Many researchers have described the deacetylase histone inhibitors as a new group of drugs that improve the longterm memory formation. These drugs can improve memory in normal animals as well as in animal with brain disorders. The objective of this study was to study the neuroprotective effects of sodium butyrate, as a histone deacetylase inhibitor, on improving cognitive functions in new born rats born of stressed mother. Materials and Methods: 15 pregnant female rats received foot shock (5 times per day and at the current intensity of $1 \mathrm{~mA}$ and frequency of $50 \mathrm{MHz}$ with 3 Minutes intervals for 2 seconds) between day 12 to day 18 of pregnancy. Infants born to these mothers were classified in three treatment groups, including Shock, shock + sodium butyrate 0.35 grams per liter, and shock + sodium butyrate 3.5 grams per liter. Infants born to mothers who were not under any shock during their pregnancy were used as the control group. To assess the working memory, Y maze test was performed on postnatal 30 and 60 days. Then, serum level of neural growth factor was measured by ELISA. Results: Periodic behaviors were significantly decreased in shock group. A significant increase in the group receiving sodium butyrate was observed compared to the control group. Besides, sodium butyrate administration increased serum level of neural growth factor. Working memory and serum level of neural growth factor in foot shock stress model were decreased. Conclusion: Administration of sodium butyrate revealed a neuroprotective effect probably via increase of neural growth factor and may improve the cognitive function of infants with prenatal stress.

\section{Key words:}

1. Pregnancy

2. Butyric Acid

3. Memory, Short-Term

4. Nerve Growth Factor

*Corresponding Author: Mohammad Amin Edalatmanesh

E-mail: amin.edalatmanesh@gmail.com 


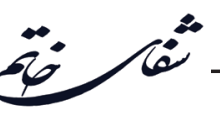

اثر سديم بوتيرات در حافظءٔ كارى و سطح بيان سرمى فاكتور رشد عصبى در مدل حيوانى با استرس در

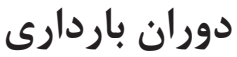

\author{
فروغ اكرمى r'، محمد امين عدالت منش'" \\ اكروه فيزيولوزى، دانشكده علوم، واحد شيراز، دانشكاه آزاد اسلامى، شيراز، ايران

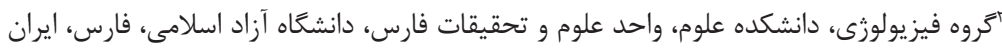

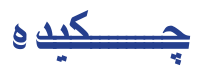

كليد وازهها:

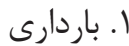

r. اسيد بوتيريك

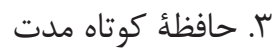

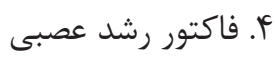

مقدمه: احساس استرس در طى باردارى شايع مىباشد و برخى زنان باردار ميزانى از سطح اضطراب

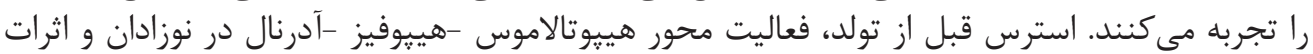

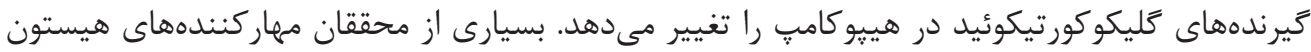

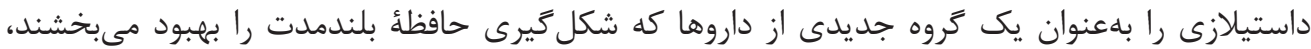

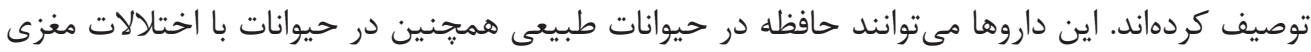

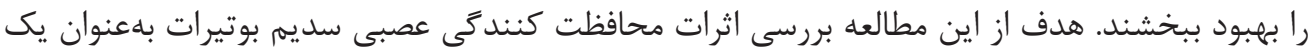

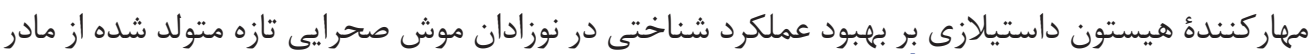

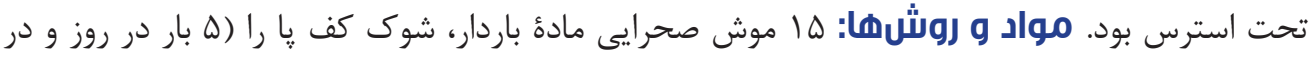

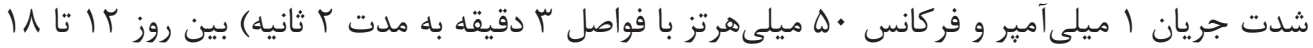

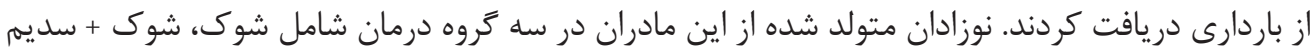

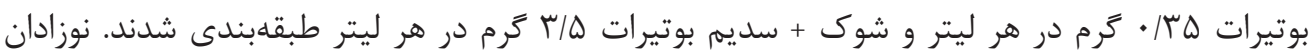

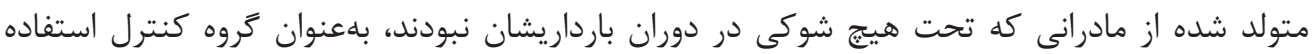

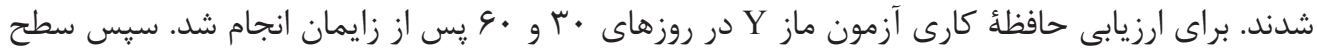

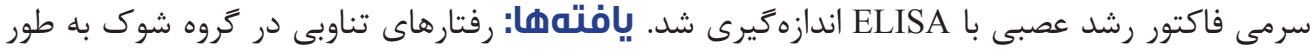

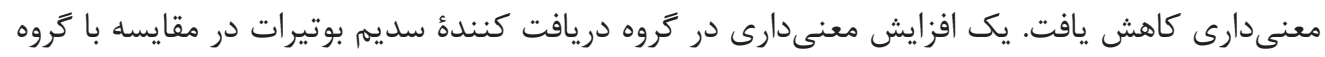

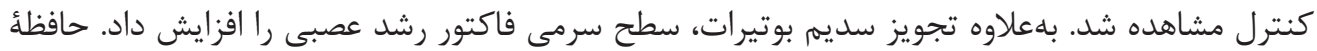

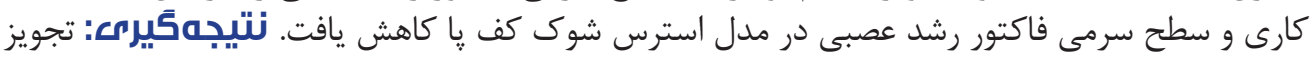

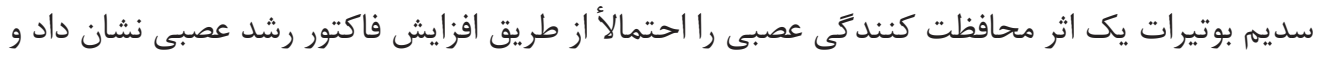

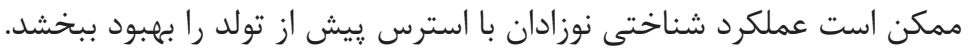

" نويسنده مسئول: محمد امين عدالت منش : آنش آدرس الكترونيكى: amin.edalatmanesh@gmail.com 


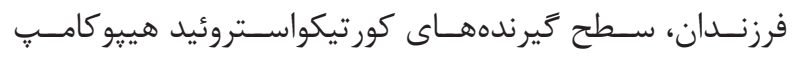

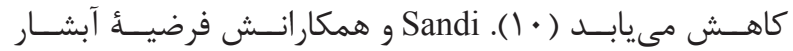

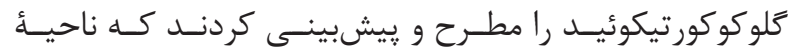

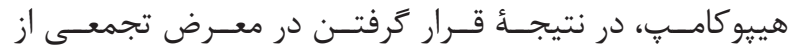

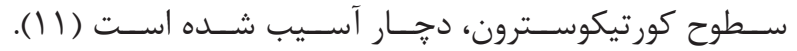

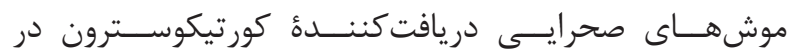

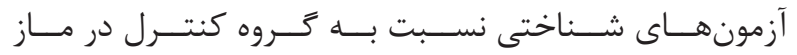

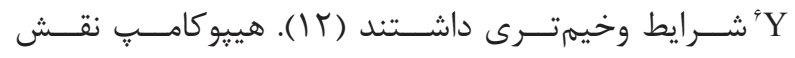

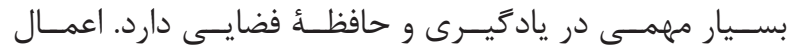

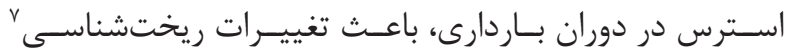

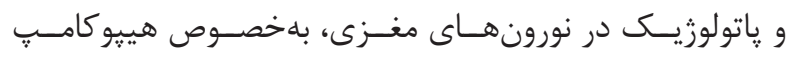

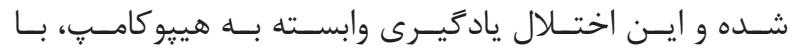

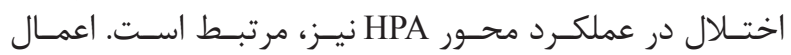

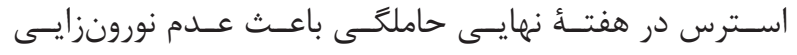

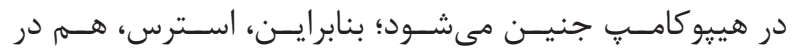

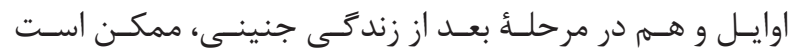

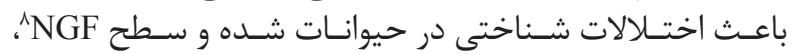

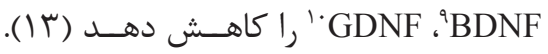

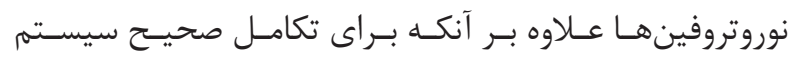

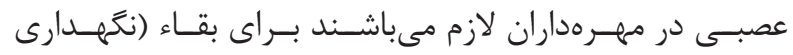

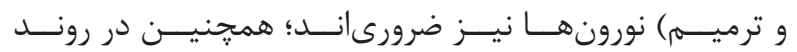

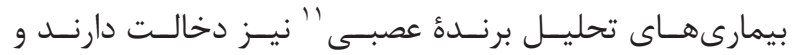

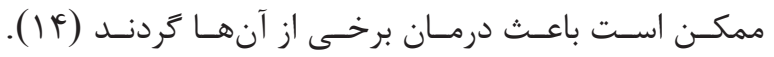

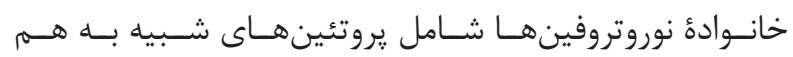

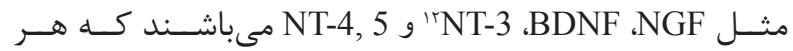

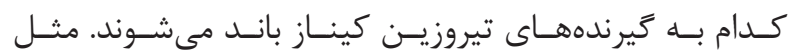

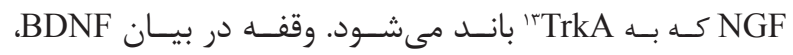
NGF

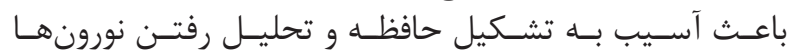

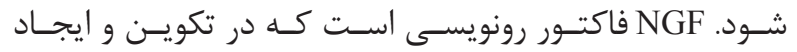

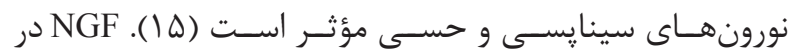

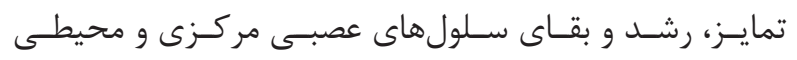

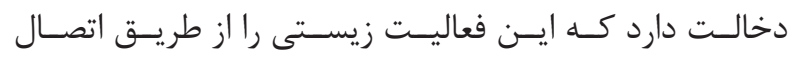

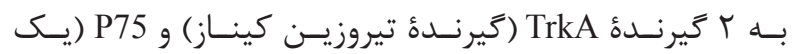

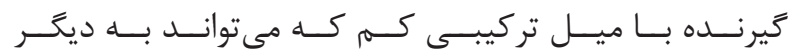

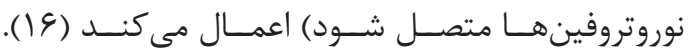

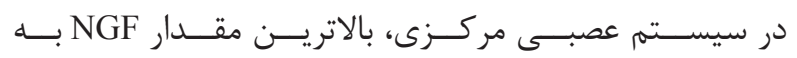

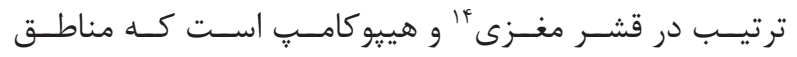

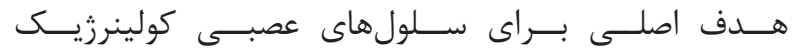

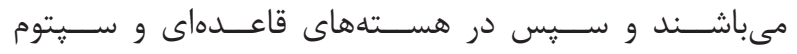

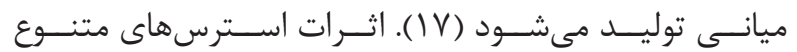

${ }^{1}$ Glucocorticoids

${ }^{2}$ Corticotropin-releasing hormone

${ }^{3}$ Hypothalamus-pituitary-adrenal

${ }^{4}$ Cholinergic

${ }^{5}$ 5-Hydroxytryptamin

${ }^{6} \mathrm{Y}$ maze

${ }^{7}$ Morphology

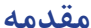

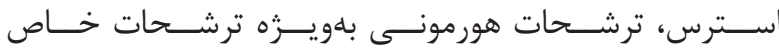

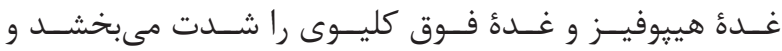

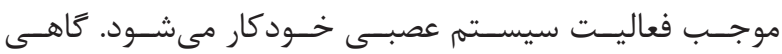

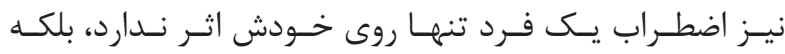

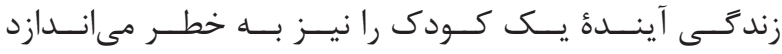

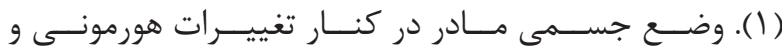

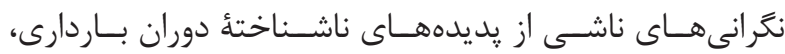

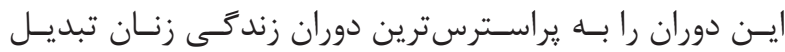

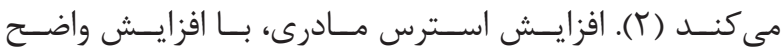

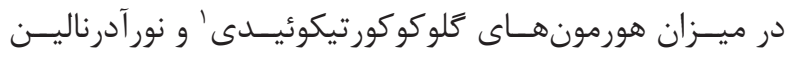

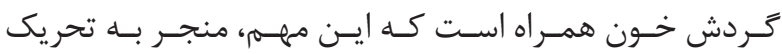

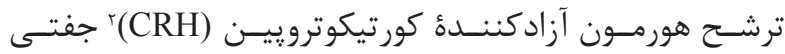

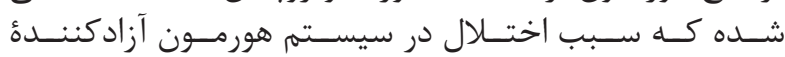

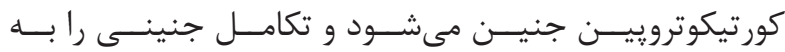

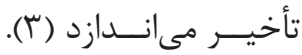

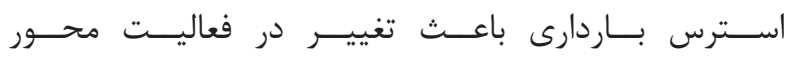

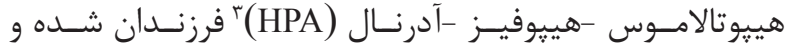

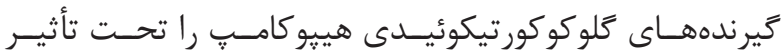

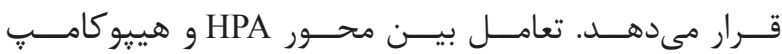

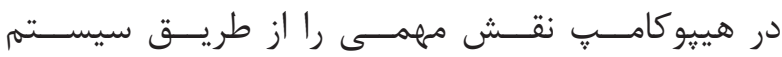

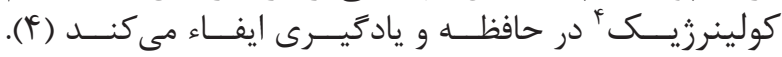

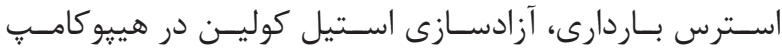

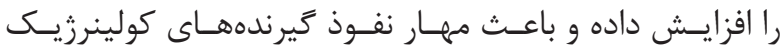

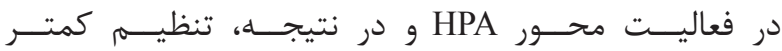

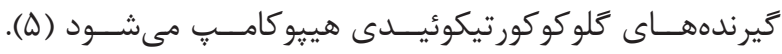

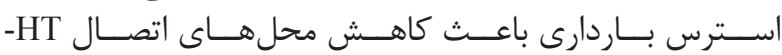

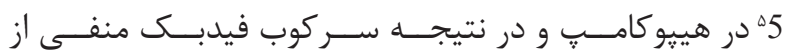

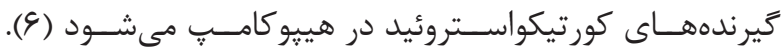

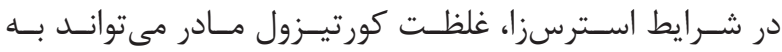

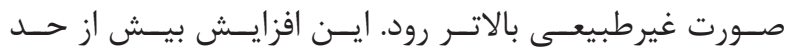

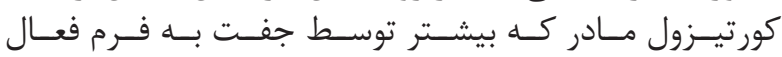

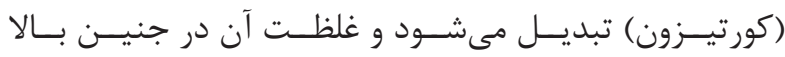

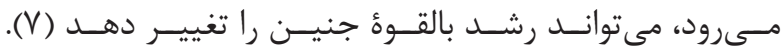

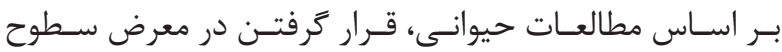

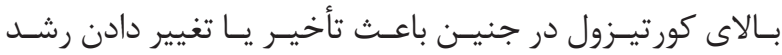

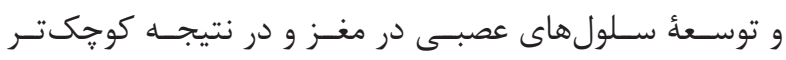

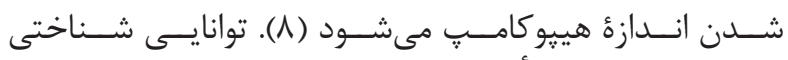

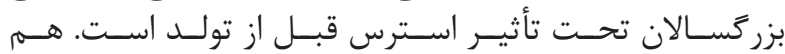

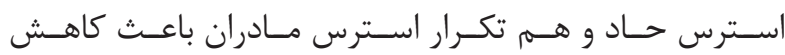

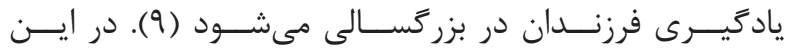

\footnotetext{
${ }^{8}$ Nerve growth factor

${ }^{9}$ Brain derived neurotrophic factor

${ }^{10} \mathrm{Glial}$ cell line-derived neurotrophic factor

${ }^{11}$ Neurodegeneration

${ }^{12}$ Neurotrophin-3

${ }^{13}$ Tropomyosin-related kinase A

${ }^{14}$ Cortex
} 


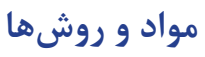

حيوانات

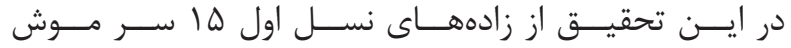

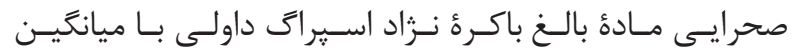

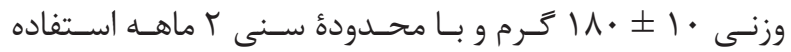

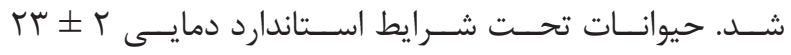

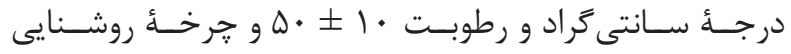

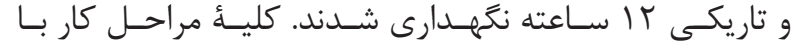

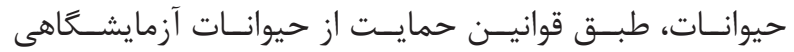

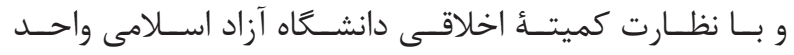

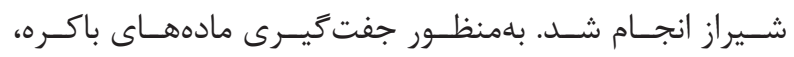

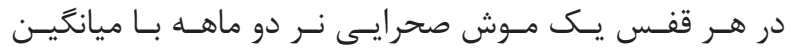

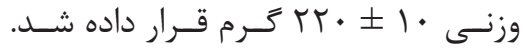

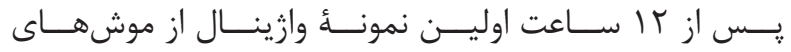

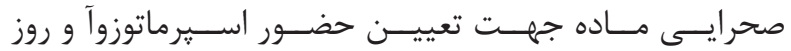

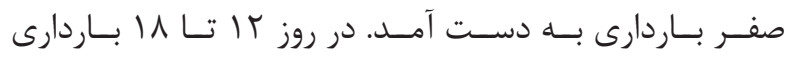

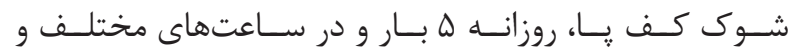

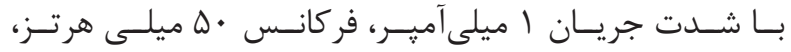

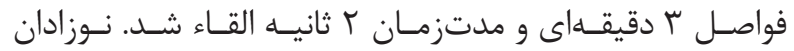

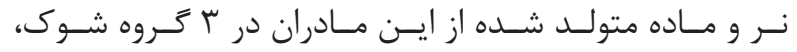

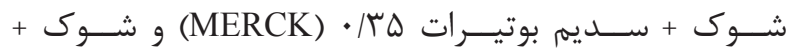

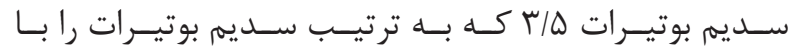

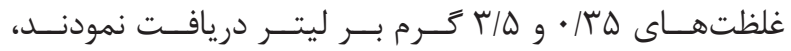

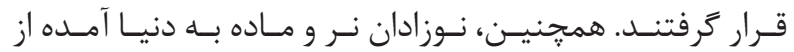

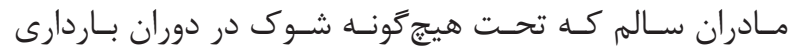

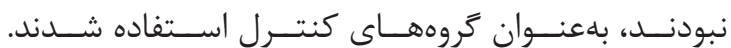

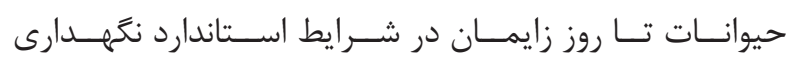

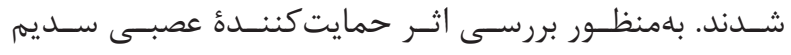

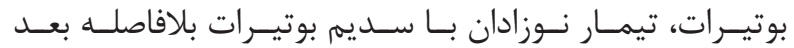

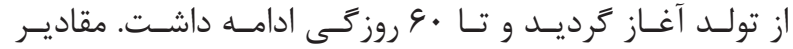

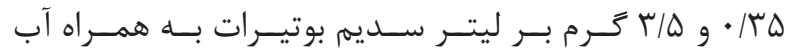

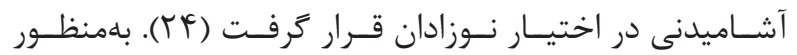

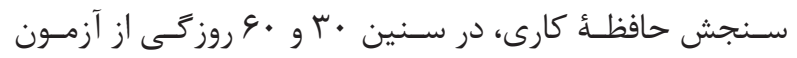

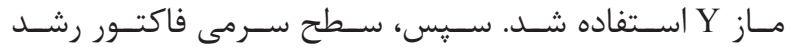

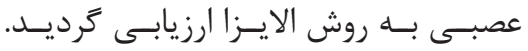

آزمون ماز Y (ارزيابى حافظةُ كارى)

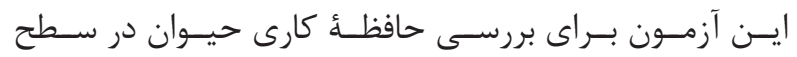

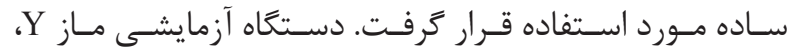

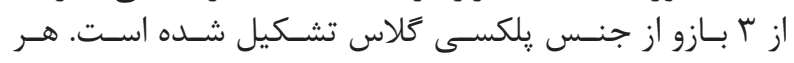

${ }^{15}$ Paradoxical sleep deprivation

${ }^{16}$ Histone deacetylase inhibitor

${ }^{17}$ Histone acetyl transferases

${ }^{18}$ Histone deacetylases

${ }^{19}$ Sodium butyrate

${ }^{20}$ Valproic acid

${ }^{21}$ Hydroxamic acids

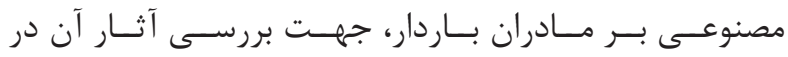

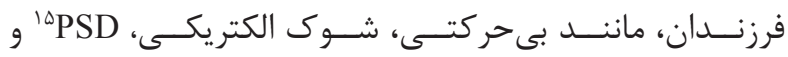

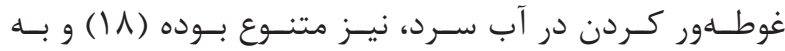

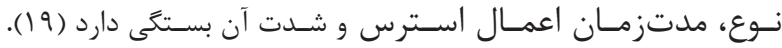

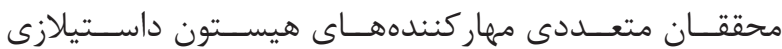
19 (HDACi)

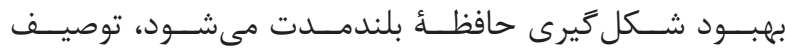

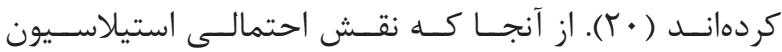

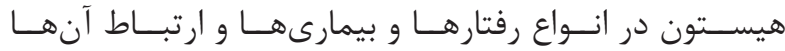

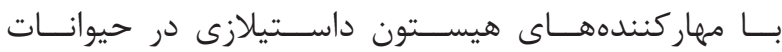

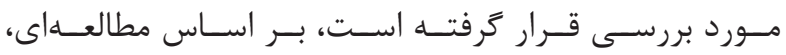

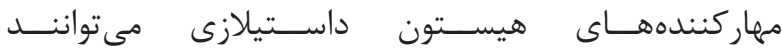

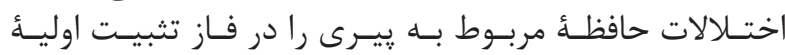

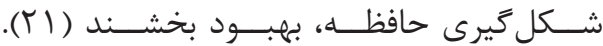

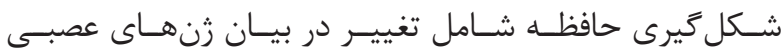

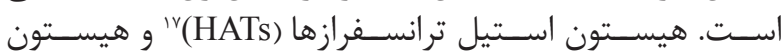

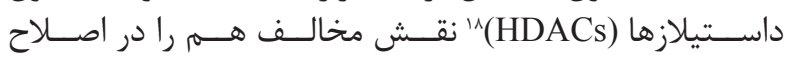

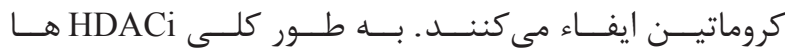

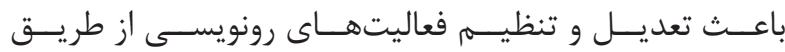

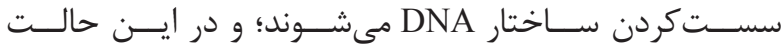

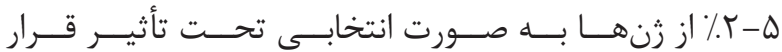

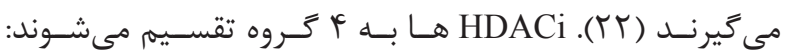

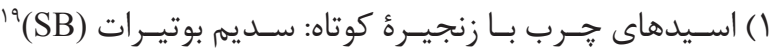

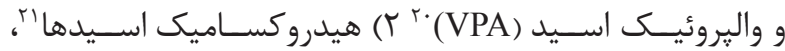

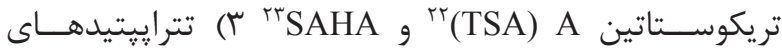

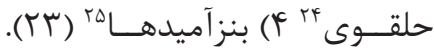

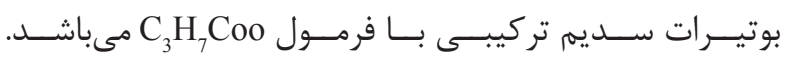

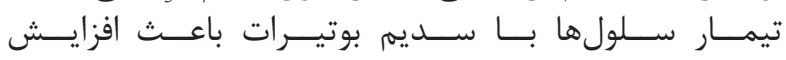

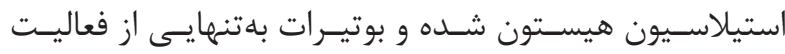

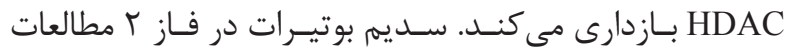

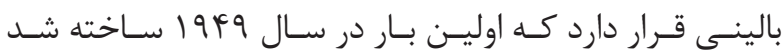

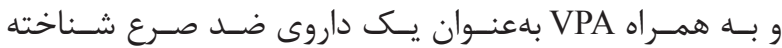

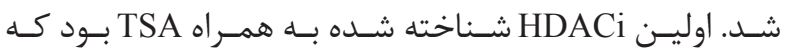

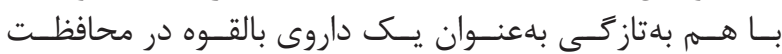

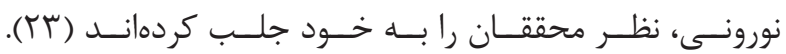

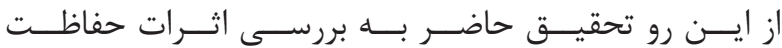

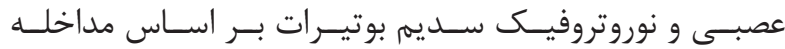

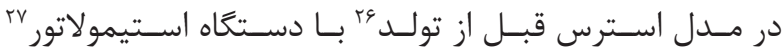

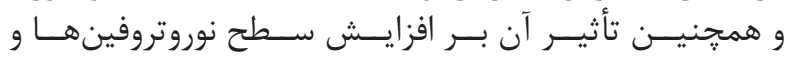

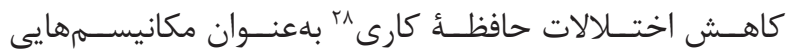

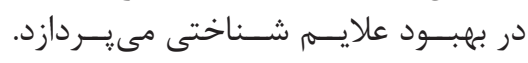

${ }^{22}$ Trichostatin A

${ }^{23}$ Suberoylanilide hydroxamic acid

${ }^{24}$ Cyclic tetrapeptides

${ }^{25}$ Benzamides

${ }^{26}$ Prenatal

${ }^{27}$ Stimulator

${ }^{28}$ Working memory 


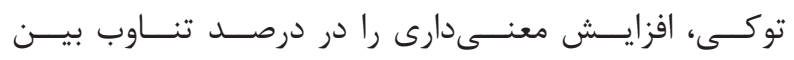

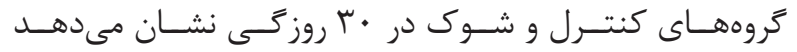
در $\left.\mathrm{F}_{r .4 \varphi}=\Lambda 9 / 4 \mid \Delta\right)$

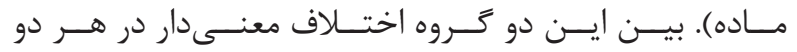

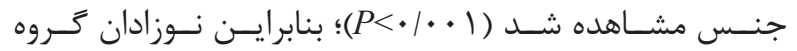

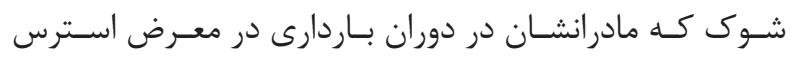

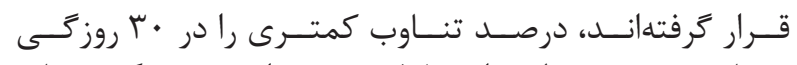

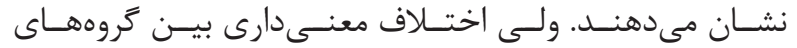

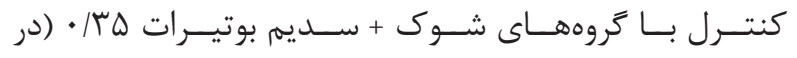

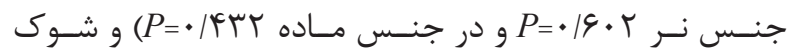

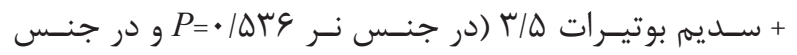

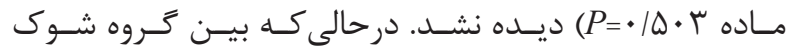

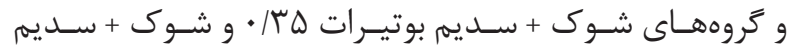

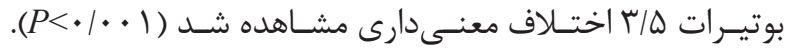

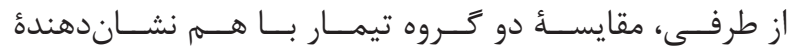

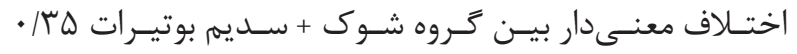

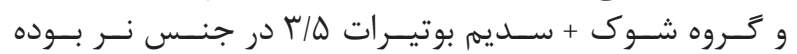

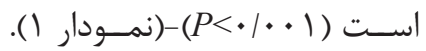

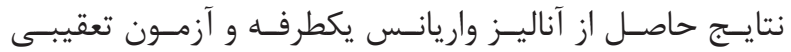

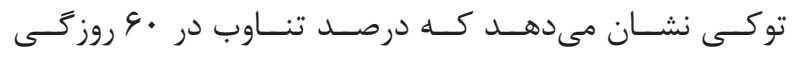

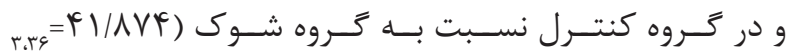

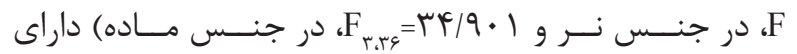

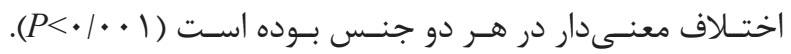

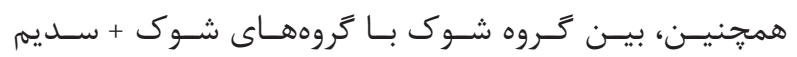

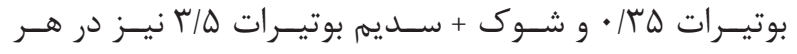

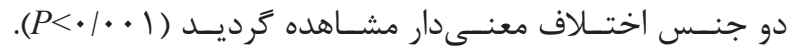

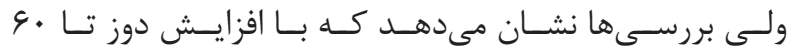

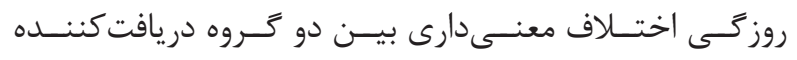

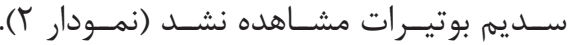

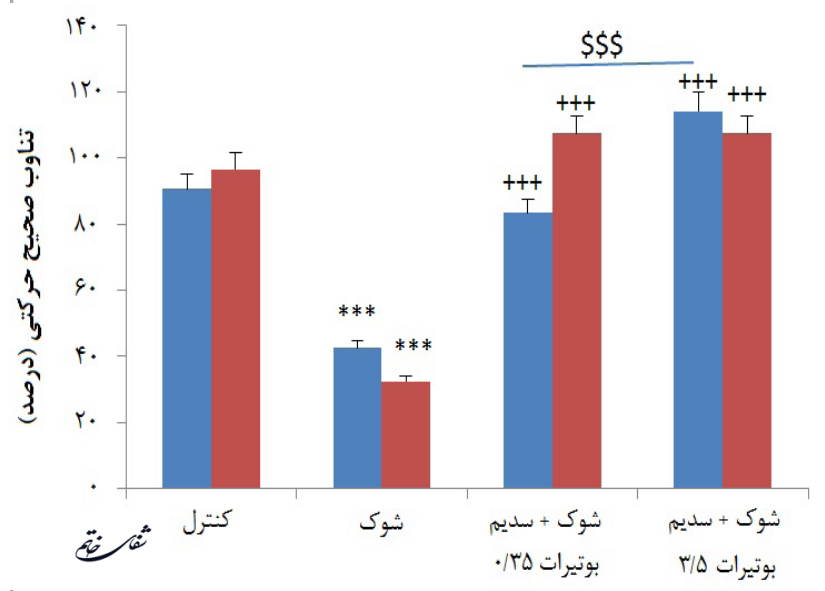

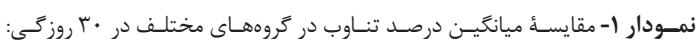

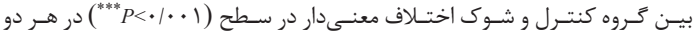

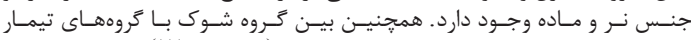

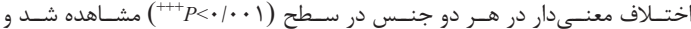

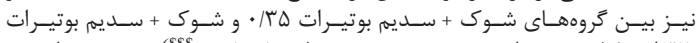

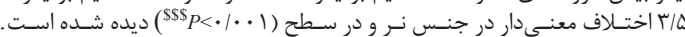

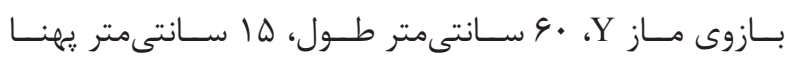

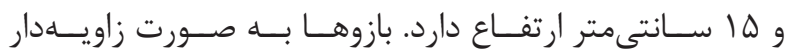

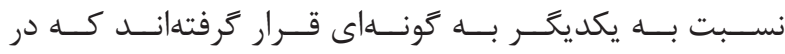

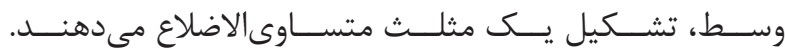

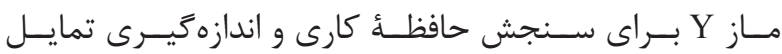

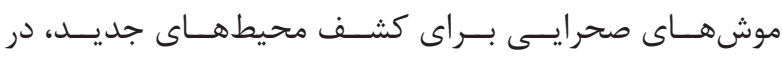

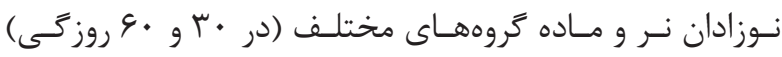

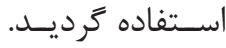

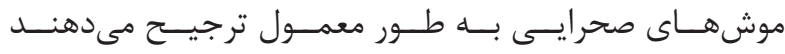

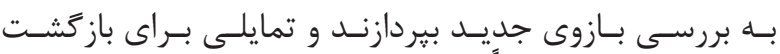

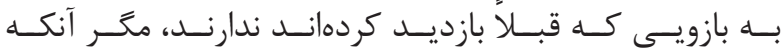

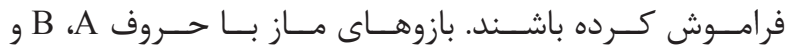

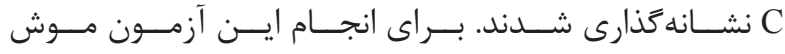

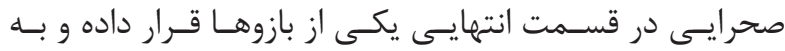

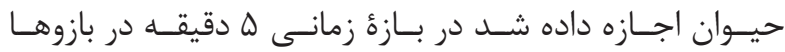

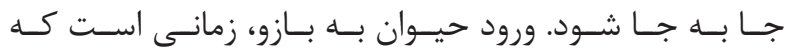

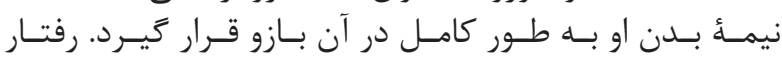

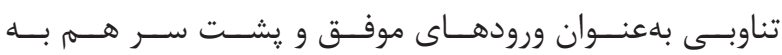

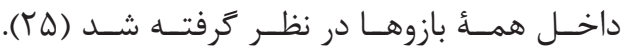

$$
\text { سنجش سطح سرمى فاكتور رشد عصبى }
$$

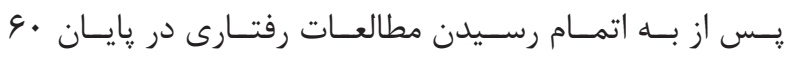

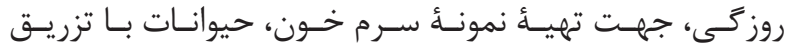

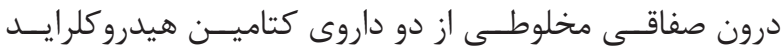

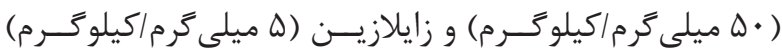

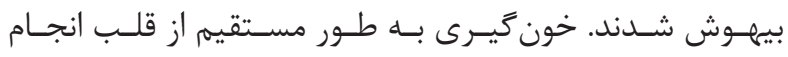

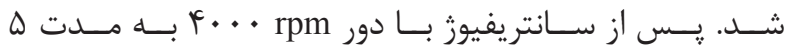

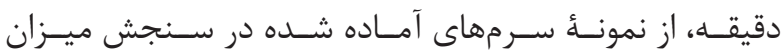

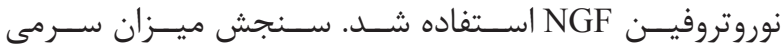

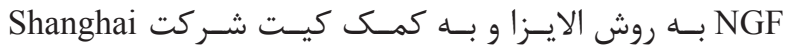

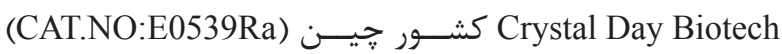

$$
\text { محاسبات آنجـام زرديـد. }
$$

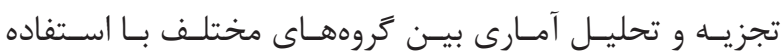

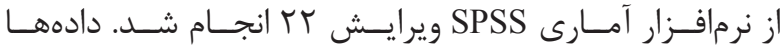

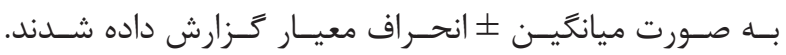

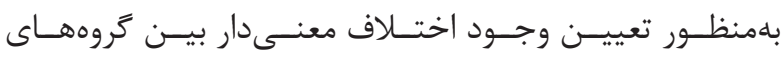

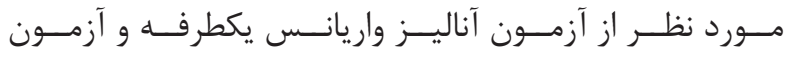

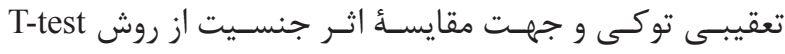

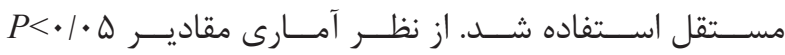
معنـى مار در نظــر زرفتـهـ شـد.

يافتهها Y آزمون ماز نتايـج حاصـل از آناليـز واريانـس يكطرفـه و آزمــون تعقيبـى 


\section{بحث و نتيجه}

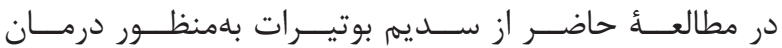

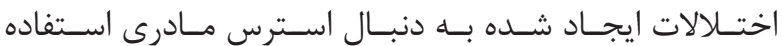

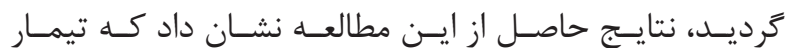

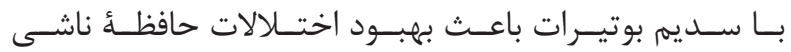

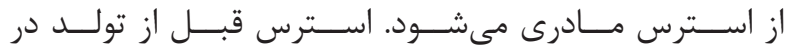

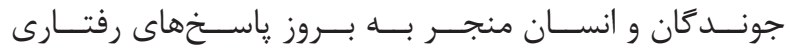

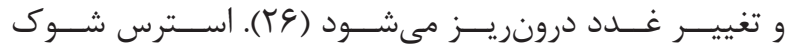

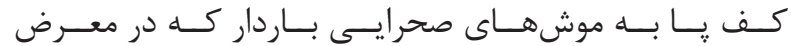

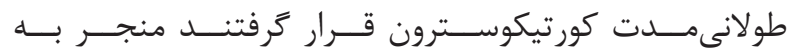

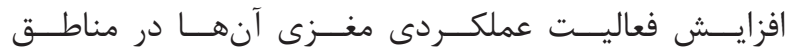

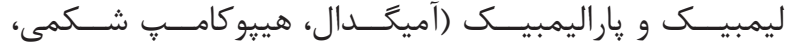

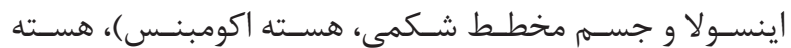

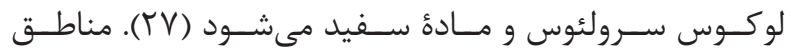

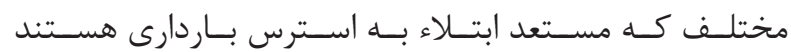

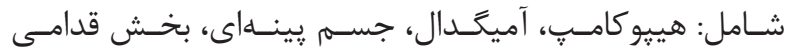

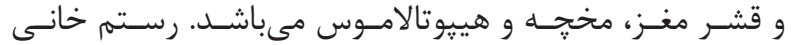

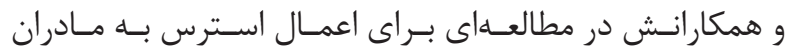

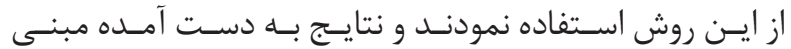

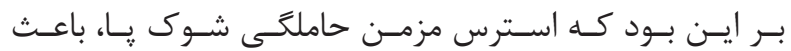

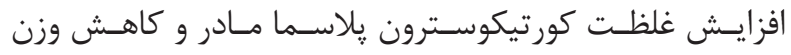

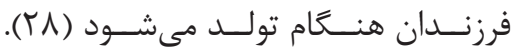

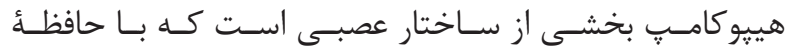

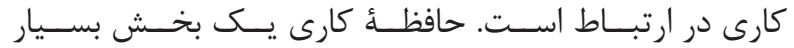

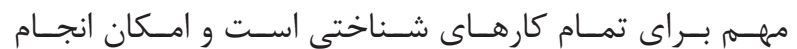

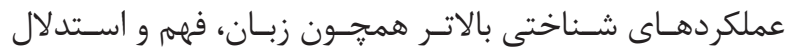

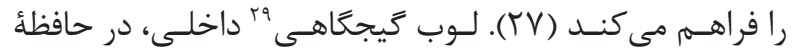

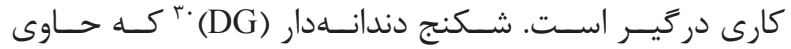

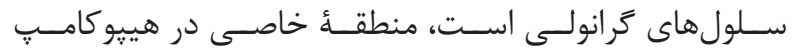

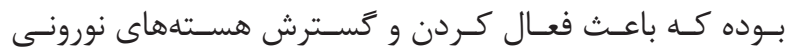

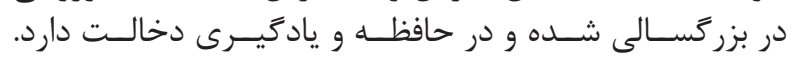

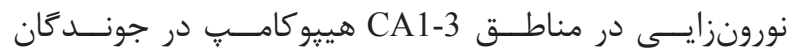

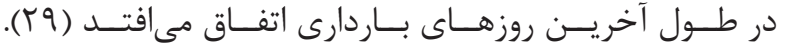

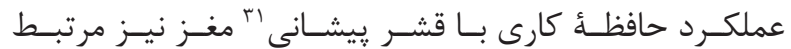

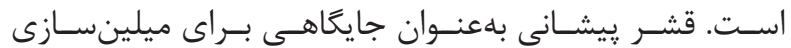

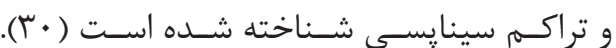

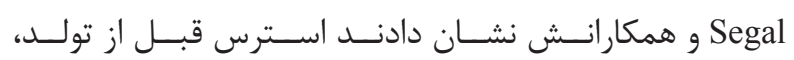

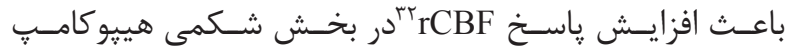

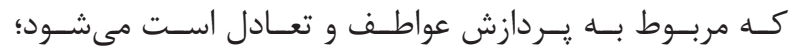

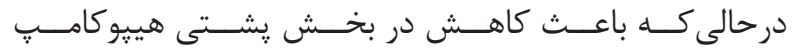

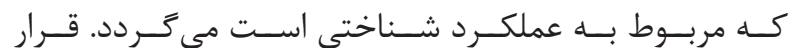

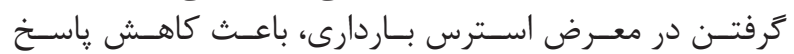
DG و CA1 CBF

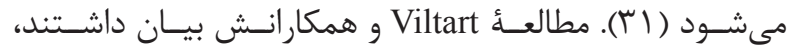

\footnotetext{
${ }^{29}$ Temporal
}

${ }^{30}$ Dentate gyrus

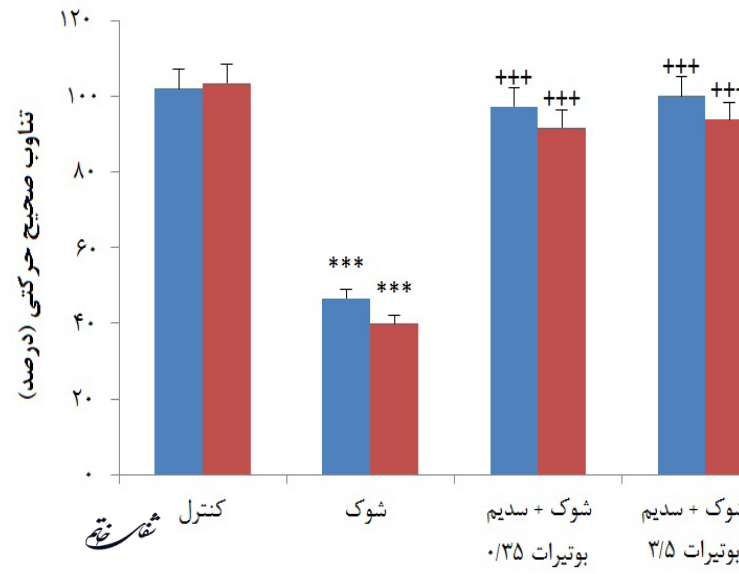

$\square$

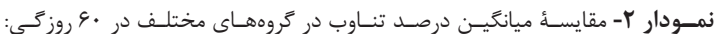

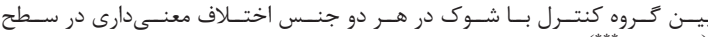

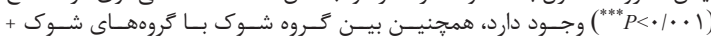

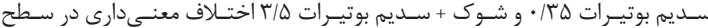

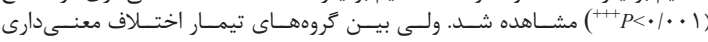
وجـود نداشت.

سنجش سطح سرمى فاكتور رشد عصبى

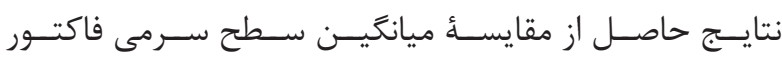

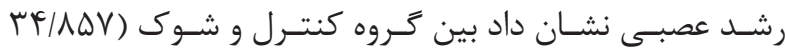

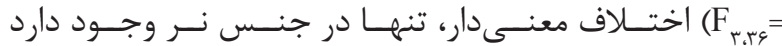

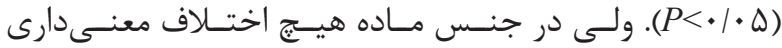

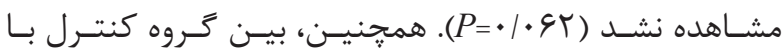

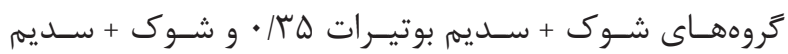

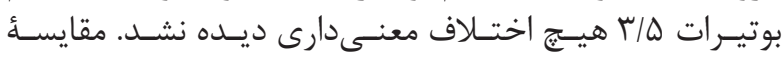

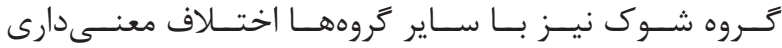

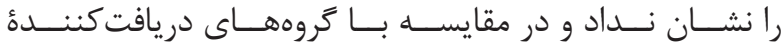

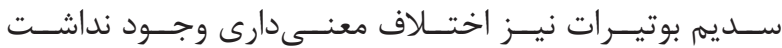

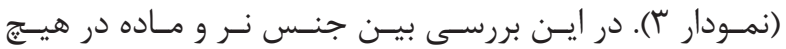

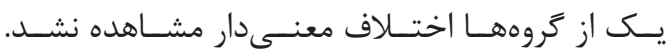

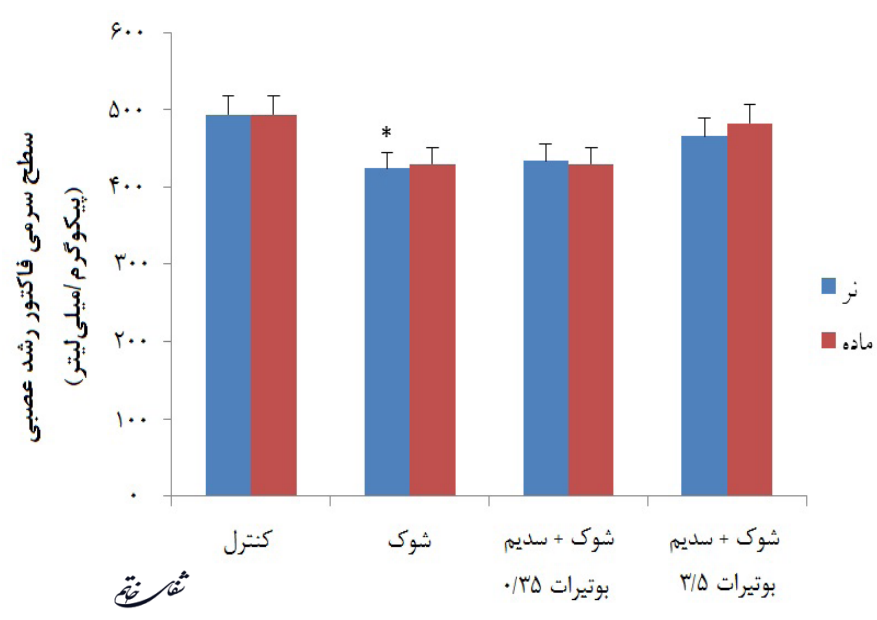

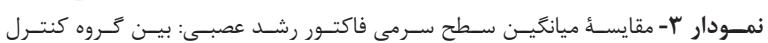

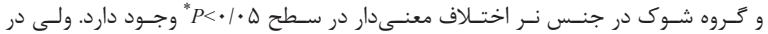

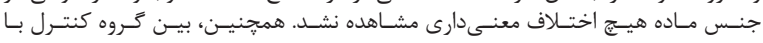

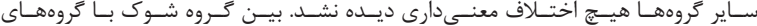

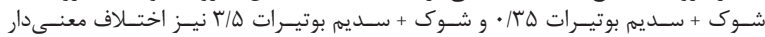

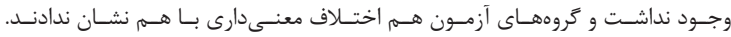

\footnotetext{
${ }^{31}$ Frontal

${ }^{32}$ Regional cerebral blood flow
} 


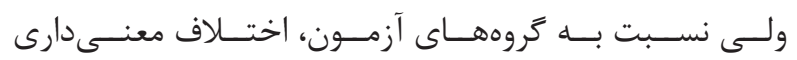

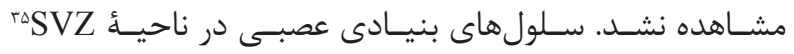

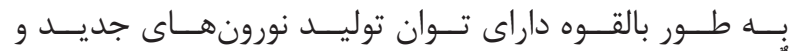

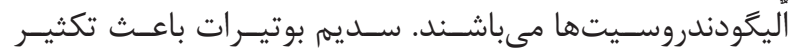

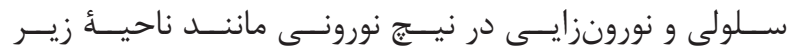

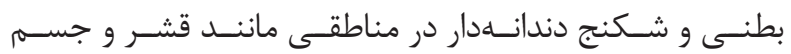

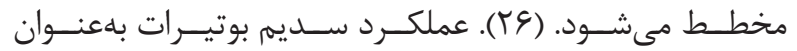

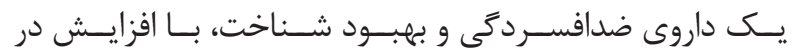

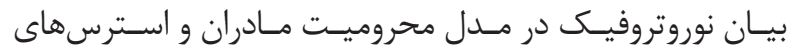

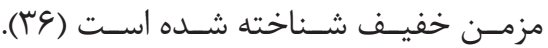

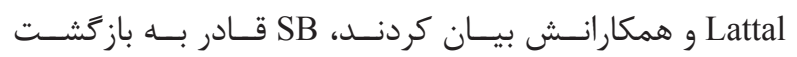

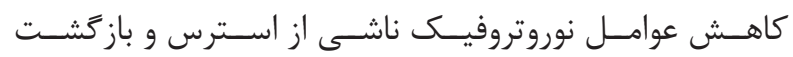

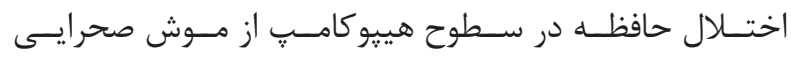

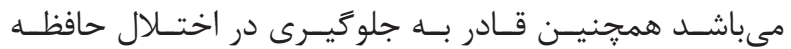

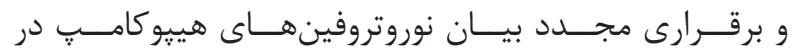

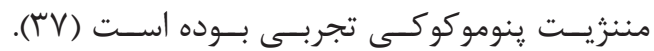

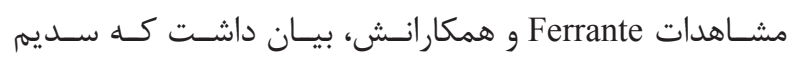

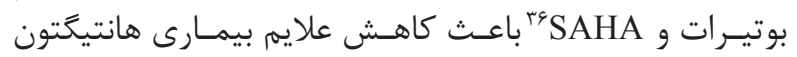

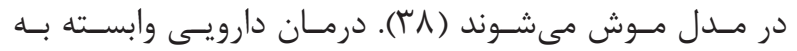

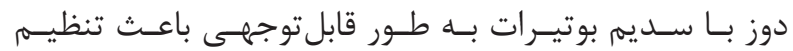

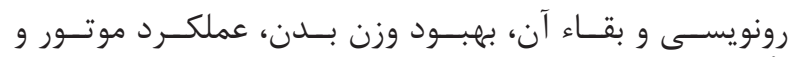

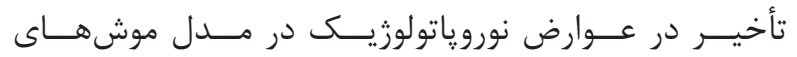

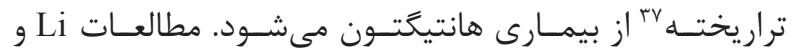

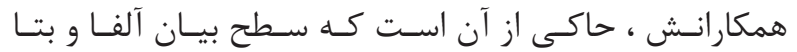

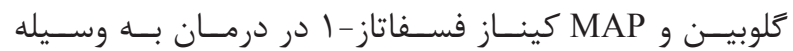

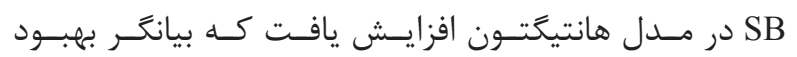

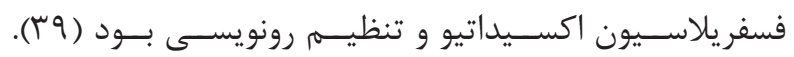

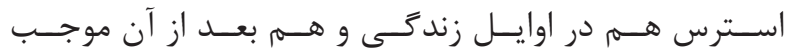

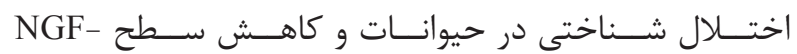

BDNF-GDNF

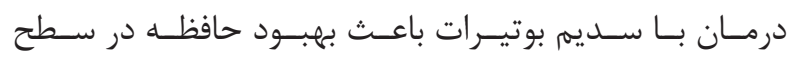

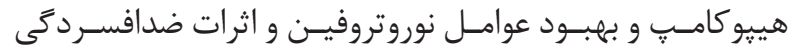

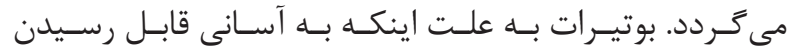

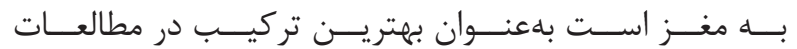

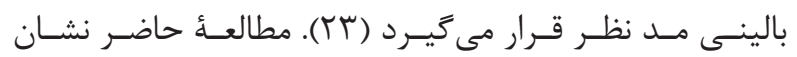

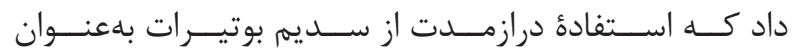

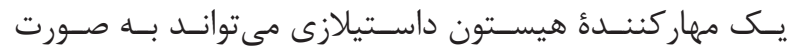

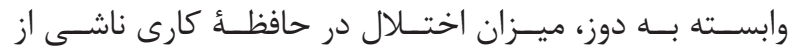

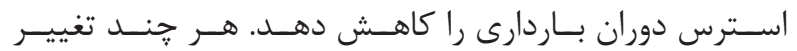

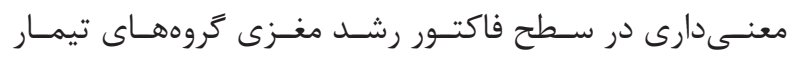

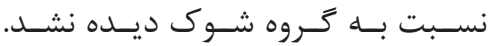

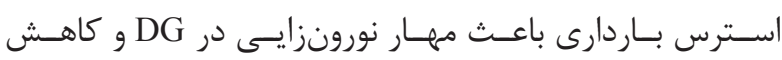

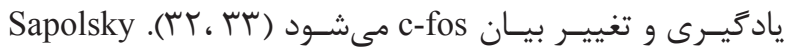

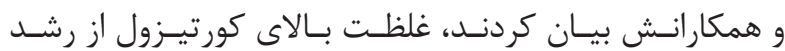

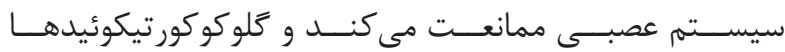

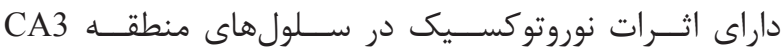

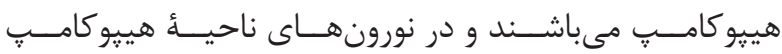

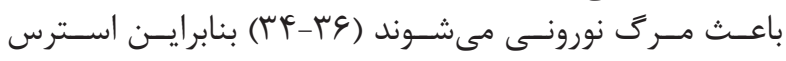

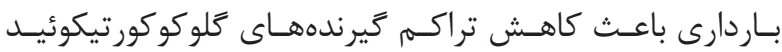

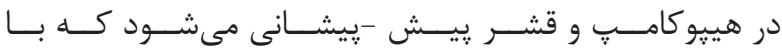

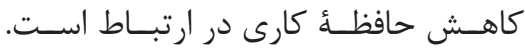

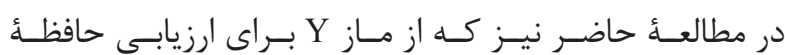

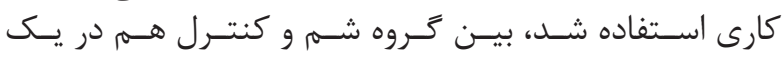

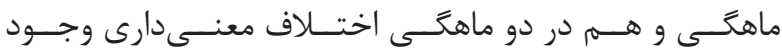

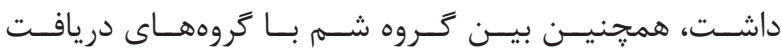

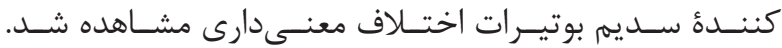

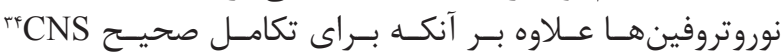

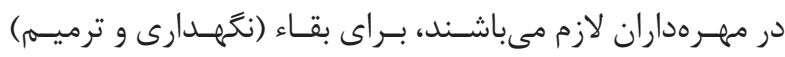

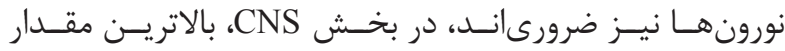
دGF

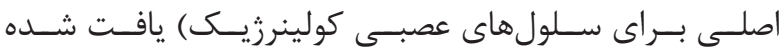

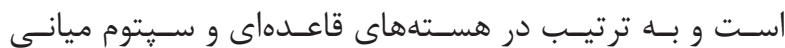

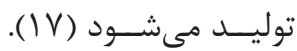

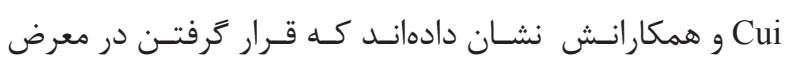

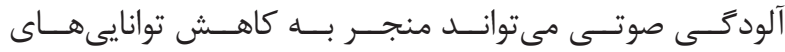

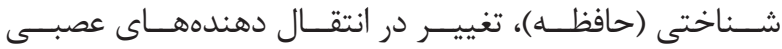

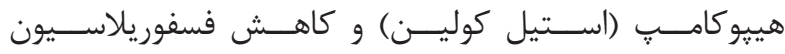

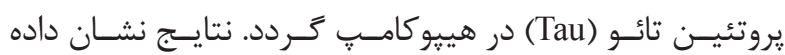

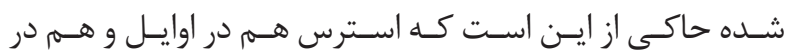

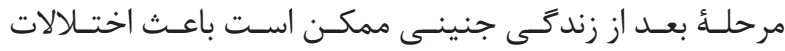

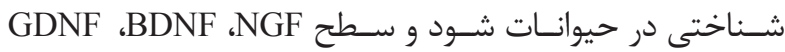

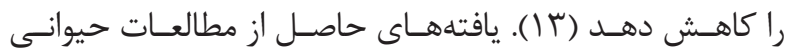

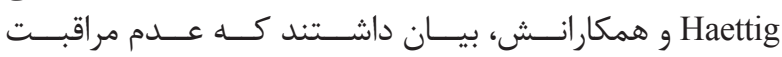

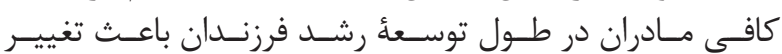

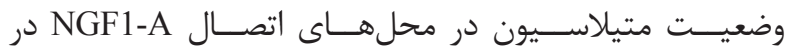

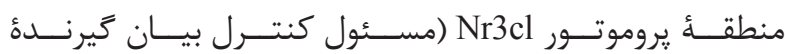

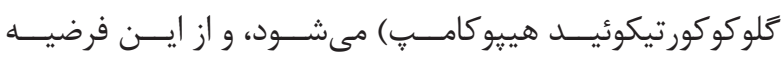

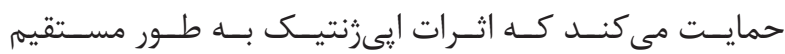

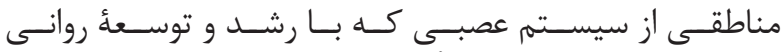

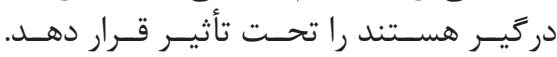

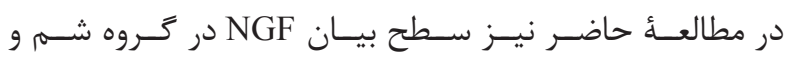

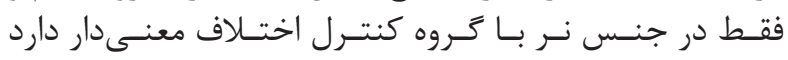

\footnotetext{
${ }^{33}$ Cerebral blood flow

${ }^{34}$ Central nervous system

${ }^{35} \mathrm{Sub}$ ventricular zone
}

\footnotetext{
${ }^{36}$ Suberoylanilide hydroxamic acid

${ }^{37}$ Transgenic
} 
1. Fuchs E, Flügge G, Ohl F, Lucassen P, VollmannHonsdorf GK, Michaelis T. Psychosocial stress, glucocorticoids, and structural alternation in the tree shrew hippocampus. Physiol Behav. 2001; 73(3): 28591

2. Corraze J, Mansour M, Dadsetan P. An outline of General Psychothology (Mental Diseases). Tehran. ROSHD Editor. 2002.

3. Mclean M, Smith R. Corticotropin releasing hormone and human parturition. Reproduction. 2001; 16(8): 493-501.

4. Messer WSJ. Cholinergic agonists and the treatment of Alzheimer's disease. Curr Top Med Chem. 2002; 2(4): $353-8$.

5. Avishai-Eliner S, Brunson KL, Sandman CA, Baram TZ. Stressed-out or in (utero)? Trends Neurosci. 2002; 25(10): 518-24.

6. Hayashi A, Nagaoka M, Yamada K, Ichitani Y, Miake Y, Okado N. Maternal stress induces synaptic loss and developmental disabilities of offspring. Int J Dev Neurosci. 1998; 16(3-4): 209-16

7. Seckl JR, Holmes MC. Mechanisms of disease:glucocorticoids, their placental metabolism and fetal'programming' of adult pathophysiology. Nat Clin Pract Endocrinol Metab. 2007; 3(6): 479-88.

8. Coe CL, Kramer M, Czéh B, Gould E, Reeves AJ, Kirschbaum C, et al. Prenatal stress diminishes neurogenesis in the dentate gyrus of juvenile rhesus monkeys. Biol Psychiatry. 2003; 54(10): 1025-34.

9. Weller A, Glaubman H, Yehuda S, Caspy T, Benuria $Y$. Acute and repeated gestational stress affect offspring learning and activity in rats. Physiol Behav. 1988; 43(2): 139-43.

10. Henry C, Kabbaj M, Simon H, LeMoal M, Maccari S. Prenatal stress increases the hypothalamo-pituitaryadrenal axis response in young and adult rats. J Neuroendocrinol. 1994; 6(3): 341-5.

11. Sandi C, Loscertales M, Guaza C. Experiencedependent facilitating effect of corticosterone on spatial memory formation in the water maze. Eur J Neurosci. 1997; 9(4): 637-42.

12. Vallée M, MacCari S, Dellu F, Simon H, Le Moal M, Mayo W. Long-term effects of prenatal stress and postnatal handling on age-related glucocorticoid secretion and cognitive performance: a longitudinal study in the rat. Eur J Neurosci. 1999; 11(8): 2906-16.

13. Cui $B$, Wu $M$, She $X$, Liu H. Impulse noise exposure in rats causes cognitive deficits and changes in hippocampal neurotransmitter signaling and tau phosphorylation. Brain Res. 2012; 1427: 35-43.

14. Friedman WJ. Neurotrophins induce death of hippocampal neurons via the p75 receptor. J Neurosci. 2000; 20(17): 6340-6.

15. Gadient RA, Cron KC, Otten U. Interleukin-1/3 and tumor necrosis factor-a synergistically stitmulate nerve growth faetor (NGE) release frotn cultured rat astrocytes. Neurosci Lett. 1990; 17: 335-40.

16. Chae $\mathrm{CH}$, Jung SL, An SH, Jung CK, Nam SN, Kim HT. Treadmill exercise suppresses muscle cell apoptosis by increasing nerve growth factor levels and stimulating phosphatidylinositol 3-kinase activation in the soleus of diabetic rats. J Physiol Biochem. 2011; 67(2): 235-41.

17. Cimino M, Cattabeni F, Di Luca M, Peruzzi G, Andena M, Tirassa P, et al. Levels of NGF, p75NGFR and ChAT immunoreactivity in brain of adult and aged microencephalic rats. Neurobiol Aging. 1996; 17(1): 137-42.

18. Velazquez-Moctezuma J, Dominguez Salazar E, Cruz Rueda ML. The effect of prenatal stress on adult sexual behavior in rats depends on the nature of the stressor. Physiol Behav. 1993; 53(3): 443-8.

19. Fujioka T, Fujioka A, Tan N, Chowdhury GM, Mouri $\mathrm{H}$, Sakata Y, et al. Mild prenatal stress enhances learning performance in the non-adopted rat offspring. Neurosci. 2001; 103(2): 301-7.

20. Bilang-Bleue A, Ulbricht S, Chandramohan Y, DeCarli S, Droste SK, Reul JM. Psychological stress increases histone $\mathrm{H} 3$ phosphorylation in adult dentate gyrus granule neurons: involvement in a glucocorticoid receptor-dependent behavioural response. Eur J Neurosci. 2005; 22: 1691-700.

21. Peleg S, Sananbenesi F, Zovoilis A, Burkhardt S, Bahari-Javan S, Agis-Balboa RC, et al. Altered histone acetylation is associated with age-dependent memory impairment in mice. Science. 2010; 328: 753-6.

22. Dash PK, Orsi SA, Moore AN. Histone deactylase 
inhibition combined with behavioral therapy enhances learning and memory following traumatic brain injury. Neuroscience. 2009; 163(1): 1-8.

23. Langley B, Gensert JM, Beal MF, Ratan RR. Remodeling chromatin and stress resistance in the central nervous system: histone deacetylase inhibitors as novel and broadly effective neuroprotective agents. Curr Drug Targets CNS Neurol Disord. 2005; 4(1): 4150 .

24. Cummins CL. Characterizing the expression of CYP3A4 and efflux transporters (P-gp, MRP1, and MRP2) in CYP3A4-transfected Caco-2 cells after induction with sodium butyrate and the phorbol ester 12-O-tetradecanoylphorbol-13-acetate. Pharm Res. 2001; 18(8): 1102-9.

25. Weinstock M, Matlina E, Maor GI, Rosen H, McEwen BS. Prenatal stress selectively alters the reactivity of the hypothalamic-pituitary-adrenal system in the female rat. Brain Res. 1992; 595(2): 195-200.

26. Haettig J, Stefanko DP, Multani ML, Figueroa DX, McQuown SC, Wood MA. HDAC inhibition modulates hippocampus-dependent long term memory for object location in a CBP-dependent manner. Learn Mem. 2011; 18(2): 71-9.

27. Baddeley AD. Working memory. New York. Oxford University Press. 1986.

28. Rostamkhani F, Zardooz H, Parivar K, Hayati Roodbari M. Prenatal stress induces metabolic impairment in adolescent male Wistar rat. Advances in Bioresearch. 2013; 4(1): 5-11.

29. Bayer SA. Development of the hippocampal region in the rat. I. Neurogenesis examined with $3 \mathrm{H}$-thymidine autoradiography. J Comp Neurol. 1980; 190(1): 87-114.

30. Yakovlev PL, Lecours AR. The myelogenetic cycles of regional maturation of the brain. Oxford. Blackwell. 1967; p. 3-70.

31. Segal M, Richter-Levin G, Maggio N. Stressinduced dynamic routing of hippocampal connectivity:
A hypothesis. Hippocampus. 2010; 20(12): 1332-8.

32. Viltart O, Mairesse J, Darnaudéry M, Louvart H, Vanbesien-Mailliot C, Catalani A, et al. Prenatal stress alters Fos protein expression in hippocampus and locus coeruleus stress-related brain structures. Psychoneuroendocrinology. 2006; 31(6): 769-80.

33. Connolly JD, Goodale MA, Menon RS, Munoz DP. Human fMRI evidence for the neural correlates of preparatory set. Nat Neurosci. 2002; 5(12): 1345 52 .

34. Sapolsky RM, Uno H, Rebert CS, Finch CE. Hippocampal damage associated with prolonged glucocorticoid exposure in primates. J Neurosci. 1990; 10(9): 2897-902.

35. Barros VG, Duhalde-Vega M, Caltana L, Brusco A, Antonelli MC. Astrocyte-neuron vulnerability to prenatal stress in the adult rat brain. J Neurosci Res. 2006; 83: 787-800.

36. Poland RE, Cloak C, Lutchmansingh PJ, McCracken JT, Chang L, Ernst T. Brain N-acetyl aspartate concentrations measured by $\mathrm{H}$ MRS are reduced in adult male rats subjected to perinatal stress: preliminary observations and hypothetical implications for neurodevelopmental disorders. J Psychiatr Res. 1999; 33(1): 41-51.

37. Lattal KM, Barrett RM, Wood MA. Systemic or intrahippocampal delivery of histone deacetylase inhibitors facilitates fear extinction. Behav Neurosci. 2007; 121(5): 1125-31.

38. Ferrante RJ, Andreassen OA, Jenkins BG, Dedeoglu A, Kuemmerle S, Kubilus JK, et al. Neuroprotective effects of creatine in a transgenic mouse model of Huntington's disease. J Neurosci. 2000; 20: 4389-97.

39. Li J, Gorospe M, Hutter D, Barnes J, Keyse SM, Liu Y. Transcriptional induction of MKP-1 in response to stress is associated with histone $\mathrm{H} 3$ phosphorylationacetylation. Mol Cell Biol. 2001; 21(23): 8213-24. 